\title{
Evolución de la Política Exterior de China para América Latina ${ }^{1}$
}

The Evolution of China's Foreign Policy for Latin America ${ }^{2}$

\author{
Jose Luis Valenzuela Alvaréz ${ }^{3}$
}

Resumen: La publicación por la República Popular China en noviembre 2016 de un nuevo Libro Blanco sobre su política para con América Latina y El Caribe, permite desarrollar un análisis comparativo que evidencia la evolución de dicha política entre los años 2008 y 2016. Este análisis comparado se complementa con la visión académica actual y otras acciones políticas desarrolladas por China en campos complementarios.

Palabras Clave: China; América Latina y El Caribe; política bilateral; política exterior de China

Summary: The publication by the People's Republic of China in November 2016 of a new White Paper on its policy towards Latin America and the Caribbean allows the development of a comparative analysis that shows the evolution of this policy between the years 2008 , the date of publication of its first Book White in this regard, and 2016. This comparative analysis is complemented by the current academic vision and other policy actions developed by China in complementary fields.

Key words: China; Latin America and The Caribbean; bilateral politics; Chinese foreign politics

\footnotetext{
1 Recibido: 19/06/17. Aceptado: 27/09/17

2 Este trabajo se basa en la tesis posdoctoral (inédita) del autor, desarrollada en la Universidad Estadual Paulista, Sao Paulo y Marilia, Brasil

3 Doctor en Estudios Americanos, Universidad de Santiago, Chile. Posdoctor en Relaciones Internacionales, Universidad Estadual Paulista, Brasil. Ingeniero Civil Químico, Universidad de Chile. Director Académico del Centro de Estudios Latinoamericanos sobre China, CELC, Universidad Andrés Bello, Chile. Email: jlvale@gmail.com
} 


\section{Introducción}

En noviembre 2016, el Gobierno de China emitió el documento titulado "Documento de Política de China sobre América Latina y El Caribe" (China's Policy Paper on Latin America and the Caribbean). (Gobierno de China, 2016).

El 11 de mayo 2008 había emitido un documento con el mismo título, (Gobierno de China, 2008), y el 20 de junio 2012 el entonces Primer Ministro de la República Popular China, Wen Jiabao pronunció un discurso en CEPAL (Santiago de Chile), que constituyó también una formulación de la política de China hacia América Latina y El Caribe, ALC. (Wen, 2012).

Aunque la política de China hacia ALC está completamente establecida en el documento más reciente, el desarrollo de un análisis comparado de los tres documentos, permite determinar su evolución.

Dado que los documentos de 2008 y 2016 tienen la estructura correspondiente a una declaración formal de política exterior, el análisis comparado se centra en ambos y se complementa con una discusión sobre el aporte del discurso-documento de 2012.

Finalmente, se revisa la literatura académica actual pertinente, y se analizan documentos recientes del gobierno de China que tienen relación con la temática principal del paper.

\section{Análisis comparado 2008 versus $\mathbf{2 0 1 6}$}

El análisis se desarrolla a partir de la preparación de documentos de comparación estructurados temáticamente. Se trata de cinco documentos de comparación, de acuerdo al ordenamiento del documento 2008. El primero se refiere a los planteamientos globales; el segundo al Área Política; el tercero al Área Económica, el cuarto al Área Social y Cultural, y el quinto al área de paz, seguridad y justicia. Se agrega el análisis de temáticas incorporadas en el documento 2016, no abordadas en el documento 2008.

El análisis busca detectar e interpretar los principales cambios en cada área temática.

\section{i. Comparación de los planteamientos globales}

\section{a) Sistema internacional}

El documento 2016 reconoce un empeoramiento del entorno económico y de las amenazas tradicionales y no-tradicionales a la seguridad, concluyendo que "Salvaguardar la paz mundial y promover el desarrollo común sigue siendo una tarea tremenda" (Gobierno de China, 2016: 1). En 2008 se afirmaba que "La multipolarización del mundo se vuelve irreversible" (Gobierno de China, 2008: 1), y en 2016, junto con afirmar que "la multi-polarización y la globalización aumentan" (Gobierno de China, 2016: 1), señala que "el surgimiento de los mercados emergentes y de los países en desarrollo se ha convertido en una tendencia histórica irresistible" (Gobierno de China, 2016: 1).

\section{b) La visión China de sí misma}

En 2016 se destaca que "China ha entrado en una etapa crucial para lograr el gran rejuvenecimiento de la nación china" (Gobierno de China, 2016: 1), y se declara que "China está dispuesta a ampliar los intereses comunes con otros países, promover la 
construcción de un nuevo tipo de relaciones internacionales con una cooperación beneficiosa para todos y forjar una comunidad de futuro compartido" (Gobierno de China, 2016: 1). En 2008 se declaraba que "China ..., está dispuesta a desarrollar la amistad y la cooperación con todos los países sobre la base de los Cinco Principios de Coexistencia Pacífica, a fin de promover la construcción de un mundo armonioso de paz duradera y prosperidad compartida" (Gobierno de China, 2008: 1)

\section{c) La visión de China sobre América Latina}

Mientras en 2008 se planteaba que "abrigando fuertes aspiraciones a la unidad y el autofortalecimiento, los países latinoamericanos y caribeños están enfrascados en la promoción de la paz, la estabilidad y el desarrollo regionales, lo que resulta en el constante robustecimiento del poderío integral y la influencia internacional de la región" (Gobierno de China, 2008: 1); afirmación que se atenúa en 2016, planteando que "América Latina y el Caribe en su conjunto cuenta con enormes potenciales de desarrollo y perspectivas brillantes, por lo que es una fuerza creciente en el panorama mundial" (Gobierno de China, 2016: 1).

\section{d) Sobre las relaciones sino-latinoamericanas}

En 2008 se señalaba que "China y América Latina y el Caribe se encuentran actualmente en fases similares de desarrollo, compartiendo las mismas tareas emanadas de éstas y el deseo común de incrementar el conocimiento mutuo y fortalecer la cooperación" (Gobierno de China, 2008: 2), y se esperaba que "de cara al futuro, los lazos sinolatinoamericanos y sino-caribeños, dotados de enormes potencialidades y promisorias perspectivas de desarrollo, aportarán sin duda mayores contribuciones a la noble causa de la paz y el desarrollo de la humanidad" (Gobierno de China, 2008: 2).

En 2016, los planteamientos cambian, reconociendo un progreso y señalando que "las relaciones han entrado en una nueva etapa de cooperación integral ... el establecimiento del Foro de China y la Comunidad de Estados Latinoamericanos y Caribeños (Foro China-CELAC) ha creado una nueva plataforma de cooperación entre ambas partes, que establece el rumbo para el desarrollo simultáneo y complementario de la cooperación bilateral y colectiva entre China y América Latina y el Caribe" (Gobierno de China, 2016: 1), y precisan que "sobre la base de la igualdad y el beneficio mutuo, la asociación amplia y cooperativa entre China y América Latina y el Caribe se orienta hacia el desarrollo común ... sirve como un ejemplo brillante de países en desarrollo que trabajan juntos para buscar un desarrollo común, asumir responsabilidades comunes y afrontar desafíos comunes en la nueva era" (Gobierno de China, 2016: 2).

\section{e) Fundamentos de la política de China hacia América Latina}

En 2008, China planteaba que "el Gobierno chino se esforzará por establecer y desarrollar con los países latinoamericanos y caribeños la asociación de cooperación integral caracterizada por la igualdad, el beneficio recíproco y el desarrollo compartido" (Gobierno de China, 2008: 2). En 2016 el planteamiento es

China se ha comprometido a construir una nueva relación con América Latina y el Caribe con cinco rasgos sobresalientes, a saber, la sinceridad y la confianza mutua en el campo político, la cooperación mutuamente ventajosa en el frente económico, el aprendizaje mutuo en cultura, Así 
como el refuerzo mutuo entre la cooperación de China con la región en su conjunto y sus relaciones bilaterales con países individuales de la región. Nuestro objetivo es acercar la alianza global y cooperativa a una nueva altura, llevando a las dos partes a una comunidad de futuro compartido en la que todos los países se unan en el desarrollo (Gobierno de China, 2016: 2).

Los objetivos eran en 2008 "- Ampliar el consenso basado en el respeto y la confianza mutuos. Ateniéndose a los Cinco Principios de Coexistencia Pacífica; - Profundizar la cooperación en el espíritu del beneficio recíproco y la ganancia compartida; - Estrechar los intercambios en aras del mutuo aprendizaje y el progreso común" (Gobierno de China, 2008: 2-3).

En 2016 se plantea que

China está dispuesta a trabajar con los países latinoamericanos y caribeños para construir el nuevo marco de cooperación pragmática "1 + $3+$ $6^{4}$ " (guiado por el Plan de Cooperación China-América Latina y el Caribe (2015-2019), utilizando el comercio, la inversión y La cooperación financiera como fuerzas motrices y la identificación de la energía y los recursos, la construcción de infraestructuras, la agricultura, la fabricación, la innovación científica y tecnológica y la tecnología de la información como prioridades de cooperación) (Gobierno de China, 2016: 2) .

Se plantea además un concepto de política internacional, "China y los países de América Latina y el Caribe comparten una responsabilidad global en la promoción de la cooperación internacional, la equidad y la justicia" (Gobierno de China, 2016: 2) , y China declara que "promoverá la multipolarización, promoverá la democracia y el estado de derecho en las relaciones internacionales y aumentará la representación y la voz de los países en desarrollo" (Gobierno de China, 2016: 2), invitando a los países ALC a acompañarla en su camino.

China afirma en 2016 que "promover el desarrollo complementario de la cooperación colectiva y bilateral es el camino estratégico para el desarrollo de las relaciones entre China y América Latina y el Caribe" (Gobierno de China, 2016: 2).

\section{ii. Comparación de los planteamientos en el área política}

\section{a) Intercambios de alto nivel.}

En 2008 se proponía "intensificar el intercambio de experiencias sobre el gobierno" (Gobierno de China, 2008: 3), lo que desaparece en el documento 2016. Sin embargo, este nuevo documento dedica un punto exclusivo al tema 'Intercambio de experiencias sobre la gobernanza', que se analiza más adelante.

\section{b) Cooperación en asuntos internacionales}

En 2008 se planteaba que "el Gobierno chino tiene la disposición de seguir fortaleciendo la coordinación y colaboración con los países latinoamericanos y caribeños en los 
asuntos internacionales, manteniendo con ellos una frecuente comunicación sobre los temas internacionales y regionales de relevancia y apoyándose mutuamente en temas trascendentales que atañan a la soberanía estatal e integridad territorial de una y otra parte" (Gobierno de China, 2008: 4). Se agregaba que "China es partidaria de que los países latinoamericanos y caribeños jueguen un papel más importante en la arena internacional" (Gobierno de China, 2008: 4).

En 2016 no se menciona este tema en el área política, el que está cubierto ampliamente en el capítulo 5 'Colaboración internacional'.

Dicho capítulo aborda los conceptos de 'asuntos políticos internacionales', 'Gobernanza económica global', 'implementación de la agenda 2030 para el desarrollo sustentable', 'respuesta al cambio climático', y 'CiberSeguridad'.

Respecto al primer concepto se plantea "profundizar los esfuerzos para los intercambios y la cooperación de ambas partes en Naciones Unidas y otras organizaciones internacionales y plataformas internacionales, mantener la comunicación y la coordinación en los principales temas internacionales y regionales, reforzar la voz de los países en desarrollo en los asuntos internacionales, y salvaguardar el interés de ambas partes y de otros países en desarrollo" (Gobierno de China, 2016: 6).

En lo referido al segundo concepto, China plantea "la coordinación y cooperación ... en las organizaciones internacionales económicas y financieras ... avanzar en el proceso de negociaciones comerciales centrado en la OMC ... integración económica en la región Asia-Pacífico" (Gobierno de China, 2016: 6) , y declara sus intenciones de "llamar a la comunidad internacional para continuar promoviendo la reforma de la gobernanza económica global ... avanzar más en la reforma del FMI ... apoyar al Banco Mundial para un mayor avance en la revisión de la participación accionaria según acuerdo de todas las partes ... promover la construcción de una red financiera global de seguridad" (Gobierno de China, 2016: 6).

Respecto al tercer concepto, China se declara "lista para trabajar con los países LAC para promover una cooperación ganar-ganar en la implementación de la Agenda 2030 para el desarrollo sustentable ... reforzar el estatus de los principales canales de cooperación Norte-Sur ... mientras se da importancia al papel de la cooperación Sur-Sur y la cooperación trilateral" (Gobierno de China, 2016: 7).

El cuarto concepto, respuesta al cambio climático, está incluido en las temáticas 2008, mientras en el quinto concepto China se declara lista para trabajar con los países ALC en "construir un ciberespacio de paz, seguridad, apertura y cooperación, un sistema de gobernanza para Internet que sea multilateral, democrático y trasparente" (Gobierno de China, 2016: 7).

\section{c) Intercambio de experiencias sobre la gobernanza}

Se trata de un tema nuevo, incluido en 2016. Se plantea que "las dos partes deben sacar enseñanzas y sabiduría de sus tradiciones históricas y prácticas de desarrollo, y fortalecer aún más los intercambios de experiencias sobre gobernanza y desarrollo, a fin de impulsar el desarrollo común" (Gobierno de China, 2016: 3), y se invita a los países ALC a "participar activamente en el Centro Internacional para el Desarrollo del Conocimiento establecido por China" (Gobierno de China, 2016: 3).

El Centro al que se hace referencia había sido anunciado por el Presidente Xi Jinping en Naciones Unidas, septiembre 2015. Manifestó que "China creará un centro de conocimiento para el desarrollo internacional en el que se intercambiarán estudios, ex- 
periencias y buenas prácticas" (ONU, 2015: Para.4). Llamado en inglés 'Center for International Knowledge on Development (CIKD)', se funda bajo el alero del Centro de Investigaciones sobre el Desarrollo (en inglés 'China's Development Research Center', DRC), un centro de pensamiento dependiente directamente del Consejo de Estado de China, el cual inicia la definición de su función principal como "realizar investigaciones avanzadas sobre temáticas globales, generales, estratégicas y de largo plazo en cuanto al desarrollo económico y social, así como sobre los problemas claves y candentes relacionados con la reforma y la apertura, ofreciendo opciones políticas y asesoramiento al Comité Central del PCCh y al Consejo de Estado" (DRC, 2017:Who does what). El CIKD constituye un paso adelante en la internacionalización del CD.

No se aprecian diferencias significativas en otros planteamientos, específicamente en 'Intercambio entre los órganos legislativos', 'Intercambio entre los partidos políticos', 'Mecanismos de consulta', y 'contacto entre los gobiernos locales'.

\section{iii. Comparación de los planteamientos en el área económica}

\section{a) Comercio}

Mientras en 2008 China ofrece "seguir esforzándose junto con los países latinoamericanos y caribeños por ampliar y equilibrar el comercio bilateral y optimizar la estructura comercial, con el objetivo de promover el desarrollo conjunto, al mismo tiempo de solucionar adecuadamente las fricciones comerciales a través de consultas y cooperaciones" (Gobierno de China, 2008: 4), en 2016 su propuesta es bastante específica, "promover el comercio de productos especializados, bienes con ventajas competitivas o de alto valor añadido, así como productos intensivos en tecnología, y fortalecer el comercio de servicios y la cooperación en comercio electrónico entre China y los países de América Latina y el Caribe" (Gobierno de China, 2016: 3). Asimismo, mientras en 2008 manifestaba su "disposición de considerar activamente, sobre la base de beneficio recíproco y ganancia compartida, la suscripción de tratados de libre comercio con los países u organizaciones de integración regional" (Gobierno de China, 2008: 5), en 2016 plantea que "sobre la base del principio de reciprocidad y beneficio mutuo, China debatirá con los países de América Latina y el Caribe el establecimiento de relaciones comerciales a largo plazo y estables, así como diversos acuerdos de facilitación comercial incluyendo el TLC" (Gobierno de China, 2016: 3).

\section{b) Inversión}

En 2008 se declaraba que "el Gobierno chino estimula y apoya a las diversas empresas chinas con capacidad y de buena reputación a desplegar la cooperación en inversión en América Latina y el Caribe" (Gobierno de China, 2008: 5), lo que en 2016 se amplía: "China apoyará los esfuerzos de las empresas chinas para invertir e iniciar negocios en los países de América Latina y el Caribe y alinear la capacidad de alta calidad y los equipos más ventajosos de China, con las necesidades de los países de América Latina y el Caribe, con el fin de ayudar a los países en necesidad a mejorar su capacidad de desarrollo independiente" (Gobierno de China, 2016: 3). China declara asimismo que "desea firmar más acuerdos sobre protección de las inversiones, evitar la doble imposición y la evasión fiscal con los países de América Latina y el Caribe, con el fin de crear un entorno y condiciones favorables para la cooperación en materia de inversiones entre empresas de ambas partes" (Gobierno de China, 2016: 3). 


\section{c) Cooperación financiera}

En 2008 China planteaba "mayor comunicación e intercambio y cooperación profesional entre las autoridades monetarias y financieras ... establecimiento de sucursales de los bancos comerciales chinos en ALC ... suscripción de acuerdos de cooperación con los países de la región en supervisión bancaria y desplegará la cooperación destinada a combatir el lavado de dinero y el financiamiento al terrorismo" (Gobierno de China, 2008: 5).

En 2016 se agrega "ampliar los arreglos transfronterizos en la moneda local, examinar los acuerdos de compensación de RMB y promover de manera constante la cooperación monetaria" (Gobierno de China, 2016: 3), y se incluye el "pleno desarrollo al papel del Fondo de Cooperación China-América Latina, los préstamos en condiciones favorables, los préstamos especiales para la infraestructura chino-latinoamericana, China y el Caribe" (Gobierno de China, 2016: 3), y se compromete a que "explorará activamente formas de cooperación, incluyendo seguros y leasing financiero, ampliará continuamente la cooperación con instituciones financieras regionales en América Latina y el Caribe, y apoyará la cooperación en áreas clave y proyectos principales entre ambas partes" (Gobierno de China, 2016: 3) .

\section{d) Cooperación agrícola}

En 2008 China mostraba su disposición a "a promover el intercambio y la cooperación con América Latina y el Caribe en tecnología agrícola y capacitación de personal ... fomentar juntos la seguridad alimentaria" (Gobierno de China, 2008: 5).

En 2016 se agrega que "China seguirá estableciendo y mejorando los programas de demostración de tecnología agrícola, promoviendo el desarrollo y demostración de tecnologías agrícolas modernas, y mejorando la innovación tecnológica agrícola, la producción y la capacidad de procesamiento agrícola, y la competitividad internacional de ambas partes" (Gobierno de China, 2016: 4) y, al mismo tiempo, se compromete a "que se dará pleno uso al papel del fondo especial para la cooperación agrícola China-América Latina" ((Gobierno de China, 2016: 4).

\section{e) Cooperación industrial}

En 2008 hay planteamientos generales como "fortalecer el intercambio ... establecer y perfeccionar los mecanismos pertinentes de cooperación, compartir sus experiencias exitosas ... promover y profundizar la cooperación práctica" (Gobierno de China, 2008: 5).

En 2016 hay propuestas mucho más concretas, entre las cuales destacan:

apoyará a sus empresas fuertes para que participen en los principales proyectos de desarrollo de recursos y energía y proyectos de construcción de infraestructura en países de América Latina y el Caribe ... construir líneas de producción y bases de servicios de mantenimiento en la región ... cooperación en campos como los automóviles, equipos de nueva energía, motocicletas y la industria química, lo que cubrirá toda la cadena industrial, para que las dos partes se pueden complementar, aumentar el empleo local, mejorar el nivel de industrialización y promover el desarrollo económico y social local (Gobierno de China, 2016: 4)

Asimismo, "se realizarán debates sobre la construcción conjunta de parques industriales, parques logísticos, parques industriales de alta tecnología, zonas económicas 
especiales y otras áreas de aglomeración industrial, para ayudar a los países latinoamericanos y caribeños en su modernización industrial" (Gobierno de China, 2016: 4), y "Los intercambios y la cooperación entre las pequeñas y medianas empresas de ambas partes se fomentarán mediante la creación de plataformas pertinentes y la creación de un buen entorno para ellos" (Gobierno de China, 2016: 4).

\section{f) Cooperación en infraestructura}

Planteamientos generales de 2008, como que China "desea reforzar la cooperación práctica con los países latinoamericanos y caribeños en la construcción de infraestructuras de transporte, información, comunicaciones, obras hidráulicas e hidroeléctricas, entre otras áreas" (Gobierno de China, 2008: 5), son sustituidos en 2016 por planteamientos concretos como "China reforzará la cooperación en la consulta técnica, la construcción y la ingeniería, la fabricación de equipos y la gestión de la operación en los campos de transporte, logística comercial, instalaciones de almacenamiento, tecnología de la información y la comunicación, energía y potencia, conservación del agua, vivienda y construcción urbana" (Gobierno de China, 2016: 4).

Se agrega "explorar activamente nuevas formas de cooperación, como la Asociación Público-Privado (PPP), con el fin de promover la conectividad de la infraestructura en América Latina y el Caribe" (Gobierno de China, 2016: 4).

\section{g) Cooperación en recursos y energías}

Se pasa nuevamente de un planteamiento general en 2008, "La parte china está dispuesta a ampliar y profundizar con los países latinoamericanos y caribeños, en el marco de los mecanismos de cooperación bilateral en los terrenos pertinentes, la cooperación mutuamente beneficiosa en materia de recursos y energías" (Gobierno de China, 2008: 6), a planteamientos específicos en 2016, como "se harán esfuerzos para llevar la cooperación a los negocios primarios como la exploración y el desarrollo, a fin de consolidar los cimientos de la cooperación y ampliar el potencial de recursos; y al mismo tiempo, la cooperación se extenderá a las industrias terciarias y de apoyo, como la fundición, el procesamiento, la logística comercial y la fabricación de equipos, a fin de mejorar el valor añadido de los productos" (Gobierno de China, 2016: 3).

"China está dispuesta a explorar ... el establecimiento de mecanismos para el suministro a largo plazo de energía y productos naturales, la fijación de precios y liquidación en moneda local para reducir el impacto de los riesgos económicos y financieros externos" (Gobierno de China, 2016: 3-4).

\section{h) Cooperación aduanera y cooperación en inspección de calidad y cuarentena}

Ambos puntos se refunden en uno solo, llamado 'Cooperación en Aduanas y Control de Calidad', de similar tenor.

\section{i) Cooperación en turismo}

Al igual que en otras temáticas, se pasa de un planteamiento general en 2008, “China desea ampliar la cooperación turística ... promoverá activamente la visita de grupos turísticos chinos a los países de la región y dará la bienvenida a los turistas provenientes de estos países" (Gobierno de China, 2008: 6), a planteamientos específicos como "apoyará la negociación de más vuelos directos entre las autoridades de aviación de 
ambas partes ... prioridad a la protección de los derechos de los consumidores de los viajeros internacionales" (Gobierno de China, 2016: 6).

\section{j) Asistencia económica y técnica}

En 2008 se plantea que "el Gobierno chino seguirá ofreciendo a los países latinoamericanos y caribeños pertinentes asistencias económicas y técnicas libres de toda condición política, y siempre que lo permitan sus posibilidades, aumentará gradualmente la asistencia en virtud de la necesidad de los países de la región" (Gobierno de China, 2008: 7). En 2016 se mantiene la oferta y se precisa que "China innovará el modelo de asistencia, dando prioridad al desarrollo de recursos humanos, planificación del desarrollo, consultoría y capacitación en materia de política económica, construcción de infraestructura, agricultura y seguridad alimentaria, reducción de la pobreza, cambio climático y asistencia humanitaria" (Gobierno de China, 2016: 5).

\section{k) Cooperación multilateral}

En 2008 se plantea "reforzar la consulta y coordinación con los países latinoamericanos y caribeños en los organismos y sistemas económicos, comerciales y financieros multilaterales para impulsar la cooperación Sur-Sur, promover el desarrollo del sistema comercial multilateral hacia una dirección más justa y razonable y ampliar el derecho a voz y a la toma de decisiones de los países en desarrollo en los asuntos comerciales y financieros internacionales" (Gobierno de China, 2008: 7). En 2016 no se incluye este punto en el área económica, sino en el área de colaboración internacional, punto 2) 'Gobernanza económica global', donde se plantea reforzar la coordinación con los países ALC en "organizaciones económicas y financieras internacionales tales como G-20, APEC, FMI, Banco Mundial y otros; además de avanzar en negociaciones multilaterales comerciales con la OMC en el centro ... promover la integración en la región Asia-Pacífico" (Gobierno de China, 2016: 6). , lo que se complementa con la promoción de reformas de organismos como el Banco Mundial y el FMI, "aumentando la representación de mercados emergentes y países en desarrollo" (Gobierno de China, 2016: 6).

En 2016 desaparece el punto 'Reducción y condonación de deudas'; y no se aprecian diferencias significativas en 'Cooperación entre las cámaras y promotoras de comercio', con lo cual termina la comparación de los puntos incluidos en 2008 en el área económica.

En 2016 aparecen puntos nuevos, los que se analizan a continuación (el número entre paréntesis corresponde a la numeración utilizada en el texto 2016, bajo el ordinal '2. In the Economic Field'):

(8) Innovación Científica y Tecnológica

(9) Cooperación Espacial

(10) Cooperación Marítima

Estos puntos plantean la cooperación científica y tecnológica entre China y los países de ALC

en campos de alta tecnología como la industria de la información, la aviación civil, la energía nuclear civil y la nueva energía, para construir más laboratorios conjuntos, centros de I + D y parques de alta tecnología ... en campos como los satélites de comunicación y de detección remota, la aplicación de datos por satélite, la infraestructura aeroespacial 
y la educación y capacitación espacial, y promoverá la aplicación de la tecnología espacial en la prevención y mitigación de desastres, vigilancia agrícola y forestal, cambio climático y otros campos ... en la ciencia y la tecnología marinas, la protección del medio ambiente marino ecológico, el cambio climático marino, la prevención y mitigación de desastres marinos y otros campos (Gobierno de China, 2016: 4).

China plantea además "el diálogo y los intercambios entre los talentos científicos y tecnológicos de ambas partes ... prestará total atención al papel de la tecnología espacial como motor del desarrollo científico, tecnológico e industrial de los países de América Latina y el Caribe" (Gobierno de China, 2016: 4).

\section{iv. Comparación de los planteamientos en el área cultural y Social}

\section{a) Intercambio cultural y deportivo}

En 2016 se mantiene el espíritu y se incrementa la especificidad, con referencias a "para poner en práctica los planes y los acuerdos de cooperación cultural firmados entre ambas partes ... 'Año del Intercambio Cultural' entre China y los países de América Latina y el Caribe ... Las dos partes ... animarán a sus atletas a participar en varios eventos deportivos celebrados en los países del otro y construirán más centros deportivos de alto nivel" (Gobierno de China, 2016: 5).

\section{b) Cooperación científica, tecnológica y educativa}

El tema ciencia y tecnología ya fue analizado. En materia educativa se plantea en 2008 "promover la firma de acuerdos de reconocimiento mutuo de diplomas y títulos académicos y aumentar el número de las becas gubernamentales a los países latinoamericanos y caribeños" (Gobierno de China, 2008: 8), mientras en 2016, bajo el título específico de 'capacitación en Educación y Recursos Humanos', se plantea que China apoyará "la capacitación de talentos especializados en las lenguas china, inglesa, española y portuguesa ... la enseñanza del idioma chino ... el desarrollo de los recursos humanos, la creación de capacidad y la cooperación en diversos campos" (Gobierno de China, 2016: 5-6).

Asimismo, en 'Intercambios académicos y de Centros de Pensamiento' se plantean los intercambios académicos y la investigación conjunta.

\section{c) Cooperación en prensa}

En 2016 se amplía hacia radio, cine, TV, Internet e industria editorial. Se incluyen propuestas concretas como "acuerdos bilaterales sobre radio, cine y televisión ... intercambio y la coproducción de programas ... intercambios de personal, así como la tecnología de los medios y la cooperación industrial ... intercambio de periodistas residentes y realizarán entrevistas conjuntas, intercambio de noticias y capacitación del personal" (Gobierno de China, 2016: 6).

\section{d) Cooperación en Protección Ambiental, Cambio Climático y Reducción de Desas- tres}

Se funden en uno solo los puntos 7, 8 y 10 de 2008. No hay cambios significativos.

\section{e) Cooperación en el alivio de la pobreza}


En 2016 se agrega "celebrar el Foro de Reducción de la Pobreza y Desarrollo de China y América Latina de manera oportuna" (Gobierno de China, 2016: 5).

\section{f) Intercambio entre los pueblos}

En 2016, bajo el título "intercambios no gubernamentales" (Gobierno de China, 2016: 6), se concretan vías de cooperación como el programa de entrenamiento 'Puente al Futuro' para mil líderes jóvenes de ambas partes, el programa de entrenamiento para jóvenes cuadros latinoamericanos.

Un tema nuevo planteado en 2016 es "gobernanza social y desarrollo social", en el cual China se declara lista para "intercambios y cooperación con los países LAC para reforzar e innovar en gobernanza social, aprender de la otra parte la experiencia en gobernanza, promover la modernización de los sistemas y la capacidad de gobernanza" (Gobierno de China, 2016: 5).

En 'cooperación en salud', 'cooperación consular', no se aprecian cambios significativos entre 2008 y 2016.

En 2016 no se incluye 'intercambio entre los pueblos'

\section{v. Comparación de los planteamientos en el área de la paz, la seguridad y la justicia}

\section{a) Intercambio y colaboración militares}

En 2016 se agrega que China "mejorará la cooperación en el comercio militar y la tecnología militar" (Gobierno de China, 2016: 7), lo que no se mencionaba en 2008.

\section{b) Cooperación en asuntos judiciales y policiales}

En 2016 se agrega que China "ampliará la cooperación en áreas como la lucha contra los crímenes, la repatriación fugitiva y la recuperación de activos ... áreas de extradición, repatriación de sospechosos de delitos, transferencia de personas condenadas y embargo, confiscación y devolución de activos criminales, coordinará las posiciones de ambas partes en la cooperación judicial multilateral internacional" (Gobierno de China, 2016: 7).

\section{vi. Cooperación Colectiva}

Nuevo en 2016, plantea que, a partir del espíritu y los objetivos principales de tres documentos, de los cuales se analizan más adelante los dos primeros:

- Declaración de Beijing de la primera reunión ministerial del Foro China-CELAC;

- Plan de Cooperación China-países ALC 2015-2019; y

- Acuerdos Institucionales y Reglas de Operación del Foro China-CELAC

"está lista para trabajar con los países LAC, sosteniendo los principios de respeto, igualdad, diversidad, beneficio mutuo, cooperación, apertura, inclusividad, e incondicionalidad, para promover activamente la cooperación en varios campos bajo el marco del Foro" (Gobierno de China, 2016: 7).

Da la bienvenida a la participación de las organizaciones regionales y las instituciones multilaterales, y anuncia que "se acomodará a las necesidades de los países menos desarrollados, los países en desarrollo sin litoral, las pequeñas islas-países en desarrollo de la región en la cooperación colectiva" (Gobierno de China, 2016: 7). 


\section{vii. Cooperación trilateral}

China se declara "lista para llevar a cabo la cooperación trilateral para el desarrollo en los países ALC, con países relevantes fuera de la región y organizaciones internacionales, bajo la premisa que tal cooperación es propuesta, acordada y dominada por países de la región" (Gobierno de China, 2016: 7). Asimismo, "alentará a sus empresas para que, sobre la base de principios comerciales, lleven a cabo cooperación trilateral en los países ALC, en los campos económico, social y cultural, con partes pertinentes" (Gobierno de China, 2016: 7). En este sentido, debe considerarse que China firmó con el Reino Unido dos acuerdos de cooperación para el desarrollo, el primero de los cuales consiste en la creación del Fondo Sino-Británico para el desarrollo de África, mientras el segundo corresponde a una Asociación Sino-Británica para el desarrollo, en el cual la parte china es representada por el DRC ya mencionado a propósito de la creación del CIKD (en 2.c) (Leach, 2015)

\section{viii. El discurso de Wen Jiabao}

El Primer Ministro de la República Popular China Wen Jiabao, en su discurso del año 2012 en CEPAL, elogia la creación de CELAC, "al aunar sus esfuerzos por la integración regional y crear la Comunidad de los Estados Latinoamericanos y Caribeños (CELAC), los países de la región han logrado incrementar enormemente su fuerza de cohesión, acción e influencia" (Wen, 2012: Para.3), y declara que "La parte china propone crear el Foro de Cooperación China-América Latina y el Caribe con vistas a instituir una plataforma de nivel más elevado para reforzar la cooperación general entre ambas partes" (Wen, 2012: Para.6). El Foro de Cooperación fue creado.

El Primer Ministro propone una serie de metas y mecanismos cuantificados, a saber, "que el volumen comercial supere los 400.000 millones de dólares en el próximo lustro" (Wen, 2012: Para.7), "la parte china se dispone a tomar la iniciativa para crear el Fondo de Cooperación China-América Latina y el Caribe, al cual instituciones financieras chinas aportarán como primer paquete 5.000 millones de dólares" (Wen, 2012: Para.7), "El Banco de Desarrollo de China liderará el otorgamiento de una línea de crédito especial de 10.000 millones de dólares, destinada a promover la cooperación en la construcción de infraestructuras, incluyendo ferrocarriles, carreteras, puertos, centrales y redes eléctricas e instalaciones de telecomunicación, entre otros sectores que tienen una estrecha vinculación con la producción y el bienestar de nuestros pueblos" (Wen, 2012: Para.7), "proponemos establecer entre China y la región un mecanismo de reserva alimentaria de emergencia de 500.000 toneladas, que se destina a contingencias naturales y a la ayuda humanitaria. El Gobierno chino aportará 50 millones de dólares para constituir el fondo especial de fomento a la cooperación agrícola entre China y la región" (Wen, 2012: Para.8), "haremos esfuerzos por lograr que, de aquí a 5 años, haya un intercambio de 500 expertos y técnicos agrícolas, y el comercio de productos agrícolas supere los 40.000 millones de dólares" (Wen, 2012: Para.8), “otorgará 5.000 becas para estudiantes latinoamericanos y caribeños en el próximo quinquenio" (Wen, 2012: Para.9).

En el terreno político, expresa que China "está dispuesta a establecer un mecanismo de diálogo periódico a nivel de Canciller entre China y la troika de la CELAC" (Wen, 2012: Para.6), que hoy funciona habiendo reemplazado la troika por un cuarteto, incluyendo así al representante de los países caribeños. Su planteamiento central es "profundizar la cooperación estratégica entre China y América Latina y el Caribe sobre la base de 
la confianza política mutua" (Wen, 2012: Para.6). La primera reunión de Cancilleres fue en Beijing, 2015, y la segunda será en Santiago, 2018.

Respecto a seguridad alimentaria, expresa que "la región reúne condiciones privilegiadas para la producción agrícola, mientras China es el mercado duradero y estable para la exportación de productos agroalimentarios de la región" (Wen, 2012: Para.8).

En un terreno de mayor proyección estratégica, el Primer Ministro propuso "la creación del Foro de Innovación Científico-Tecnológica China-América Latina y el Caribe, con vistas a reforzar la cooperación en aeronáutica, astronáutica, nuevas energías, recursos, medio ambiente, mar e investigación científica polar" (Wen, 2012: Para.9).

\section{Resultados derivados del análisis comparado}

En un mundo cada vez más complejo, con una tendencia irreversible al surgimiento de los mercados emergentes y los países en desarrollo, China se encuentra en una etapa crucial para lograr su gran rejuvenecimiento, y plantea promover la construcción de un nuevo tipo de relaciones internacionales con una cooperación beneficiosa para todos y forjar una comunidad de futuro compartido.

Considera que ALC es una fuerza creciente en el panorama mundial, y que el Foro China-CELAC ha creado una nueva plataforma de cooperación entre ambas partes, que establece el rumbo para el desarrollo simultáneo y complementario de la cooperación bilateral y colectiva entre China y América Latina y el Caribe.

Para China, la asociación amplia y cooperativa entre China y América Latina y el Caribe se orienta hacia el desarrollo común, y está basada en la sinceridad y la confianza mutua en el campo político, la cooperación mutuamente ventajosa en el frente económico, y el aprendizaje mutuo en cultura.

La asociación de cooperación integral caracterizada por la igualdad, el beneficio recíproco y el desarrollo compartido, buscada en 2008 , se visualiza hoy como acercar la alianza global y cooperativa a una nueva altura, llevando a las dos partes a una comunidad de futuro compartido en la que todos los países se unan en el desarrollo.

En estos planteamientos destacan dos conceptos:

- La confianza mutua en el campo político; y

- una comunidad de futuro compartido

Existe hoy una hoja de ruta que no existía en 2008, construida en tres documentos de alta relevancia ya mencionados:

- Declaración de Beijing de la primera reunión ministerial del Foro China-CELAC;

- Plan de Cooperación China-países ALC 2015-2019; y

- Acuerdos Institucionales y Reglas de Operación del Foro China-CELAC.

La Declaración de Beijing establece que el Plan de Cooperación China-países ALC 2015-2019 "será implementado bajo los principios de flexibilidad y participación voluntaria, de conformidad con las políticas nacionales ... y su implementación no afectará ningún programa de cooperación bilateral previamente acordado por alguna de las partes" (Foro China-CELAC, 2015a: 3).

En tanto, el Plan de Cooperación China-países ALC 2015-2019 es bastante concordante con el Libro Blanco 2016. Establece metas cuantitativas como "trabajar en conjunto para incrementar el comercio en ambos sentidos entre China y la región y de forma balanceada y mutuamente beneficiosa, a los 500 mil millones de dólares y elevar el stock 
de las inversiones recíprocas por lo menos a los 250 mil millones de dólares durante los próximos diez años" (Foro China-CELAC, 2015b: III-1); y líneas de trabajo de alto interés para la parte latinoamericana, como "intensificar la colaboración para promover la industrialización de productos de valor agregado en los Estados miembros de la CELAC" (Foro China-CELAC: V-5); "estudiar las iniciativas para la construcción conjunta de parques industriales, ciencia y tecnología, zonas económicas especiales y parques de alta tecnología entre China y los Estados miembros de la CELAC, en particular en actividades de investigación y el desarrollo, con el fin de mejorar la inversión industrial y la formación de cadenas industriales de valor" (Foro China-CELAC: VII-1); y "Fortalecer los mecanismos inter-gubernamentales de diálogo y cooperación científico - tecnológicos entre China y los Estados miembros de la CELAC" (Foro China-CELAC Id: VII-4).

Finalmente, hay una invitación hacia los países LAC para acompañar a China en una política internacional conjunta basada en promover la multipolarización, la democracia y el estado de derecho en las relaciones internacionales, y aumentar la representación y la voz de los países en desarrollo.

Sobre estas bases, hay avances considerables en la profundización de la cooperación sino-latinoamericana-caribeña, entre los cuales resaltan los siguientes:

En el área política:

- Fortalecer aún más los intercambios de experiencias sobre gobernanza y desarrollo, a fin de impulsar el desarrollo común;

- La cooperación de ambas partes en Naciones Unidas y otras organizaciones internacionales y plataformas internacionales;

- Coordinación y cooperación ... en las organizaciones internacionales económicas y financieras;

- Promover una cooperación ganar-ganar en la implementación de la Agenda 2030 para el desarrollo sustentable;

- Construir un ciberespacio de paz, seguridad, apertura y cooperación, un sistema de gobernanza para Internet que sea multilateral, democrático y trasparente.

En el área económica:

- Promover el comercio de productos especializados, bienes con ventajas competitivas o de alto valor añadido, así como productos intensivos en tecnología, y fortalecer el comercio de servicios y la cooperación en comercio electrónico entre China y los países de América Latina y el Caribe;

- Debatir con los países de América Latina y el Caribe el establecimiento de relaciones comerciales a largo plazo y estables;

- Ayudar a los países en necesidad a mejorar su capacidad de desarrollo independiente;

- Crear un entorno y condiciones favorables para la cooperación en materia de inversiones entre empresas de ambas partes;

- El pleno desarrollo al papel del Fondo de Cooperación China-América Latina, los préstamos en condiciones favorables, los préstamos especiales para la infraestructura chino-latinoamericana, China y el Caribe;

- Mejorando la innovación tecnológica agrícola, la producción y la capacidad de procesamiento agrícola, y la competitividad internacional de ambas partes;

- Construir líneas de producción y bases de servicios de mantenimiento en la región; 
- Cubrir toda la cadena industrial, para que las dos partes se pueden complementar, aumentar el empleo local, mejorar el nivel de industrialización y promover el desarrollo económico y social local;

- Construcción conjunta de parques industriales, parques logísticos, parques industriales de alta tecnología, zonas económicas especiales y otras áreas de aglomeración industrial, para ayudar a los países latinoamericanos y caribeños en su modernización industrial;

- Explorar activamente nuevas formas de cooperación, como la Asociación PúblicoPrivado (PPP), con el fin de promover la conectividad de la infraestructura;

- La cooperación se extenderá a las industrias terciarias y de apoyo, como la fundición, el procesamiento, la logística comercial y la fabricación de equipos, a fin de mejorar el valor añadido de los productos;

- Promover activamente la visita de grupos turísticos chinos a los países de la región;

- China innovará el modelo de asistencia, dando prioridad al desarrollo de recursos humanos, planificación del desarrollo, consultoría y capacitación en materia de política económica, construcción de infraestructura, agricultura y seguridad alimentaria, reducción de la pobreza, cambio climático y asistencia humanitaria;

- Cooperación científica y tecnológica en campos de alta tecnología como la industria de la información, la aviación civil, la energía nuclear civil y la nueva energía, para construir más laboratorios conjuntos, centros de I + D y parques de alta tecnología ... en campos como los satélites de comunicación y de detección remota, la aplicación de datos por satélite, la infraestructura aeroespacial y la educación y capacitación espacial, y promoverá la aplicación de la tecnología espacial en la prevención y mitigación de desastres, vigilancia agrícola y forestal, cambio climático y otros campos ... en la ciencia y la tecnología marinas, la protección del medio ambiente marino ecológico, el cambio climático marino, la prevención y mitigación de desastres marinos y otros campos;

- Total atención al papel de la tecnología espacial como motor del desarrollo científico, tecnológico e industrial de los países de América Latina y el Caribe.

En el área cultural y social:

- Mayor especificidad en la cooperación deportiva y cultural;

- capacitación de talentos especializados en las lenguas china, inglesa, española y portuguesa ... la enseñanza del idioma chino ... el desarrollo de los recursos humanos, la creación de capacidad y la cooperación en diversos campos;

- Intercambios académicos e investigación conjunta;

- Programa de entrenamiento "Puente al Futuro" para mil líderes jóvenes de ambas partes, el programa de entrenamiento para jóvenes cuadros latinoamericanos;

- Reforzar e innovar en gobernanza social, aprender de la otra parte la experiencia en gobernanza, promover la modernización de los sistemas y la capacidad de gobernanza.

En el área de paz, seguridad y justicia:

- Cooperación en el comercio militar y la tecnología militar;

- Cooperación en áreas como la lucha contra los crímenes, la repatriación fugitiva y la recuperación de activos ... áreas de extradición, repatriación de sospechosos de delitos, transferencia de personas condenadas y embargo, confiscación y devolución de activos criminales, coordinará las posiciones de ambas partes en la cooperación judicial multilateral internacional; 


\section{La discusión académica en 2016}

En la discusión latinoamericana sobre las relaciones con China, hay tres temas que sobresalen en la agenda académica de la región.

El primero de ellos es la comprensión de China sobre su efecto en los países latinoamericanos. Haibin Niu da algunas luces sobre el aprendizaje de China: "los temores a una desindustrialización originada en la competencia de China, además de una caída en la demanda de China produjeron un escenario complejo para que los países ALC repensaran su relación con la superpotencia asiática ... China actualizó su estrategia económica hacia la región enfatizando la inversión, el financiamiento y la cooperación en capacidad industrial, además del comercio" (Niu, 2016: 40). Agrega también algunos elementos para orientar la mirada de América Latina hacia China: "China no es solamente un comprador de recursos naturales, sino también un importante socio para el desarrollo, con alta tecnología y capital" (Niu, 2016: 43); y "la estrategia económica de China respecto a la cooperación en capacidad industrial ha sido diseñada para enfrentar dos desafíos: 1) internamente, China necesita encontrar modos de que su capacidad industrial doméstica vaya al mundo; y 2) externamente, necesita enfrentar los temores de desindustrialización de los países latinoamericanos, ayudándolos a desarrollar su propia capacidad industrial" (Niu, 2016: 43).

Benjamín Creutzfeldt a su vez, piensa que en América Latina, "las tensiones sociales y políticas se han visto agudizadas por el reciente auge de los ingresos que ha beneficiado a la industria extractiva y, en alguna extensión, a sectores de la agricultura, reduciendo la atención de los gobiernos al sector manufacturero, y enriqueciendo a las elites nacionales" (Creutzfeldt, 2016: 34).

Niu agrega que "para asegurar una relación sino-latinoamericana sostenible en el tiempo, es también importante evitar rivalidades estratégicas entre China y Estados Unidos en el área" (Niu, 2016: 49), lo que lleva al segundo tema.

Este es el triángulo China-América Latina-Estados Unidos. Creutzfeldt plantea que en América Latina, "la ambigua relación con potencias externas presentes en la región, y la carencia de cohesión nacional y regional, han resultado en una inadecuada comprensión de, y de respuesta a, la nueva y creciente presencia de China, después de un siglo de dominación de Estados Unidos" (Creutzfeldt, 2016:25). Asimismo, "la política de China hacia América Latina no necesariamente refleja la identidad de la región. A la inversa, la importancia que algunos países dan a la relación con China no siempre encuentra eco al otro lado del Pacífico, y detrás de este escenario se asoma la presencia de la hegemonía del Norte, con una larga historia de un involucramiento cercano con su tradicional 'patio trasero'" Creutzfeldt, 2016: 28).

Este autor concluye que "Ios ideales de la política externa de Estados Unidos democracias, economías orientadas al mercado, paz y prosperidad- son más probables de ser logrados en conjunto con las propuestas chinas de avance tecnológico, la construcción de una infraestructura sólida y de cara al futuro, y la cooperación mutuamente beneficiosa" Creutzfeldt, 2016: 38).

Un tercer tema es la capacidad latinoamericana como interlocutor de China. Al respecto, Enrique Dussel es bastante escéptico:

La capacidad institucional de ambas partes, incluyendo a los chinos, para profundizar y mejorar el conocimiento de su contraparte es extremada- 
mente débil. Este es un severo impedimento para promover la relación ya que ni China ni ningún país latinoamericano ha evaluado extensamente la relación. Al igual que en otras regiones, América Latina ha sido incapaz de entender y responder sistemáticamente a las implicaciones del activo sector público de China en el exterior, y su capacidad de proponer paquetes incluyendo proyectos llave en mano y una combinación de servicios- como ningún otro país en el mundo (Dussel, 2015: 22)

Dussel concluye que "los países latinoamericanos tienen aún gran necesidad de desarrollar una detallada agenda de cooperación con China para el corto-medio o el largo plazo. Sin un plan como este, será un gran desafío desarrollar un modelo al nivel regional para las relaciones sino-latinoamericanas" (Dussel, 2015: 22-23). Una agenda de cooperación detallada, especialmente para el largo plazo, necesita inicialmente contar con un diagnóstico claro acerca de quién es y qué busca el cooperante (China) y quién es y qué busca el cooperado (América Latina). Al respecto, Dussel señala que "aún mientras China está clara y sistemáticamente ilustrando su estrategia hacia América Latina, la región carece de las inversiones y el compromiso para desarrollar su propio entendimiento del sistema político de China, y las iniciativas en comercio, inversión y educación. Esto dificulta el entendimiento bilateral" (Dussel, 2015: 23).

\section{Conclusiones}

En un entorno más político que el de 2008, China ofrece a los países de América Latina y El Caribe el dar forma a una relación orientada hacia el desarrollo de todos los pueblos involucrados, con el norte de construir una comunidad de futuro compartido.

Propone también una actuación conjunta en los foros internacionales, orientada hacia incrementar la influencia del mundo en desarrollo en la institucionalidad financiera y comercial vigente desde el fin de la Segunda Guerra Mundial.

El planteamiento incluye los problemas que China desea solucionar (por ejemplo, la repatriación fugitiva y la recuperación de activos, la seguridad alimentaria).

Es notable la profundización de la cooperación económica en relación a lo planteado en 2008, destacando la apertura hacia el desarrollo conjunto de ciencia y tecnología, incluyendo campos de alta tecnología y con mención especial de la tecnología espacial y sus aplicaciones, la construcción de parques industriales de alta tecnología, la cobertura de toda la cadena industrial, incluyendo la industria terciaria y la industria de apoyo, el desarrollo de las personas y la capacitación de los jóvenes.

Se concluye que la nueva política internacional de China para América Latina y $\mathrm{EI}$ Caribe abre las puertas para una profundización de las relaciones que signifique un avance esencial en el camino para la construcción de un mejor futuro. Esto es muy relevante considerando que hasta ahora ninguno de los tres motores del "marco de cooperación $1+3+6$ ", esto es, el comercio, la inversión y el financiamiento, ha mostrado ser un aporte vital para el desarrollo de América Latina y El Caribe.

Por otra parte, China ha comprendido las limitaciones que presenta la relación con América Latina por la vía de CELAC, y se abre a desarrollar no solo las relaciones bilaterales, sino también relaciones trilaterales que involucren a ALC, China y un tercer país $u$ organización que sea propuesta por ALC. Puede esperarse que sean viables las relaciones 
entre China y grupos de países latinoamericanos que tengan objetivos comunes concretos en tópicos que son del también parte del interés de China.

Al mismo tiempo, es imposible eludir las carencias de América Latina y El Caribe para lograr un pleno aprovechamiento de las posibilidades ofrecidas. Es responsabilidad de los países latinoamericanos y caribeños superar estas carencias propias para aprovechar la oportunidad que China significa en cuanto a la construcción de un futuro real.

\section{Bibliografía}

Creutzfeldt, B. (2016, enero-junio). China and the U.S. in Latin America. Revista Científica General José María Córdova. Estudios Militares. Volumen 14 №17, pp. 23-40. Disponible en http://www.scielo.org.co/pdf/recig/v14n17/v14n17a03.pdf

DRC. Development Research Center. Centro de Investigaciones sobre el Desarrollo. Visto en junio 10, 2017. Disponible en http://en.drc.gov.cn/

Dussel, E. China's Evolving Role in Latin America: Can It Be a Win-Win? El papel en evolución de China en América Latina: ¿Puede ser ganar-ganar? Atlantic Council, septiembre 2015, disponible en http://www.atlanticcouncil.org/publications/reports/china-s-evolving-role-inlatin-america-can-it-be-a-win-win

Foro China CELAC. Declaración de Beijing de la Primera Reunión Ministerial del Foro CELACChina. Beijing, 8 y 9 de enero 2015a. Disponible en http://www.itamaraty.gov.br/images/ed_integracao/docs_CELAC/DECLBEIJ.20 15ESP.pdf

Foro China CELAC. Plan de Cooperación de los Estados Latinoamericanos y Caribeños - China (2015-2019). Beijing, enero 23, 2015. Disponible en http://www.chinacelacforum.org/esp/zywj_4/t1230945.htm

Gobierno de China. Texto íntegro del Documento sobre la Política de China hacia América Latina y el Caribe. Publicado en español por el Diario del Pueblo el 5 de noviembre 2008, 12 páginas. Disponible en http://spanish.peopledaily.com.cn/31621/6527840.html

Gobierno de China. Full text of China's Policy Paper on Latin America and the Caribbean. Texto íntegro del Documento sobre la Política de China hacia América Latina y el Caribe. Publicado en inglés por la Agencia Xinhua el 24 de noviembre 2016, 8 páginas en inglés. Disponible en http://en.people.cn/n3/2016/1124/c900009146474.html

Leach, Melissa. UK-China collaboration on global development: alternative golden era? Institute of Development Studies, octubre 29, 2015. Disponible en http://www.ids.ac.uk/opinion/uk-china-collaboration-on-global-developmentalternative-golden-era

Niu, Haibin. A New Era of China-Latin America Relations. Anuario de la Integración Regional de América Latina y el Caribe, No. 11, 2015, febrero 10, 2016, disponible en http://www.cries.org/?p=3076 
ONU. China anuncia fondo de asistencia Sur-Sur y condonación de deuda a países menos desarrollados. Centro de Noticias ONU, septiembre 26, 2015. Disponible en http://www.un.org/spanish/News/story.asp?NewsID=33393\#.WTwNwmjys2w

Wen, Jiabao. Para Siempre Amigos de Confianza Mutua. Discurso pronunciado por el Primer Ministro Wen Jiabao en CEPAL, Santiago de Chile, el 26 de junio 2012 (actualizado el 20 de junio 2012 a las 08:23). Disponible en http://spanish.peopledaily.com.cn/31617/7858709.html 